\title{
Protocol
}

\section{Autologous chondrocyte transplantation: a phase 1 study protocol to validate the safety and feasibility of a new advanced cell therapy product for articular cartilage repair in Brazil}

\author{
Alessandro Rozim Zorzi*, Eliane Antonioli, Moises Cohen, Camila Cohen Kaleka, \\ Andrea Tieme Kondo, José Mauro Kutner, Mario Ferretti
}

Hospital Israelita Albert Einstein, São Paulo, Brazil

Received: 22 February 2020

Accepted: 01 April 2020

\section{*Correspondence:}

Dr. Alessandro Rozim Zorzi,

E-mail: alessandro.zorzi@einstein.br

Copyright: ( ) the author(s), publisher and licensee Medip Academy. This is an open-access article distributed under the terms of the Creative Commons Attribution Non-Commercial License, which permits unrestricted non-commercial use, distribution, and reproduction in any medium, provided the original work is properly cited.

\begin{abstract}
Background: Membrane-assisted autologous chondrocyte transplantation is considered the gold standard surgical technique to treat greater than two millimetres diameter cartilage lesions in the knee in patients after conservative treatment failure. However, this technique is only available in developed countries of North America, Europe and Japan. According to Brazilian law, it is considered an advanced cell therapy product. There is currently no product of this type enabled for clinical use in Brazil. Following the request of the Brazilian regulatory agency (ANVISA), this phase 1 study was developed. The objective is to access feasibility and safety of a new membrane-assisted autologous chondrocyte product.

Methods: Three participants with a larger than two millimetres articular cartilage lesion in the distal femur or the patella, which did not improve their symptoms with conservative treatment, will be submitted to an arthroscopically assisted cartilage biopsy. After isolation and expansion in a good manufacturing practices facility, chondrocyte seeded collagen membranes will be surgically inserted in the lesion and fixed with fibrin glue. The follow-up period will last 1 year. Primary outcome will be incidence and severity of complications according to NCI-CTCAE version 4.0. Secondary outcomes will be Western-Ontario McMaster Universities Osteoarthritis Index scale, International Knee Documentation Committee subjective scale and magnetic resonance observation of cartilage repair tissue magnetic resonance scale.

Conclusions: This study, together with previous preclinical results and international experience, will allow patients in Latin America to have access to this advanced cell therapy.

Trial Registration: Brazilian registry of clinical trials RBR-6fgy76 (http://www.ensaiosclinicos.gov.br/rg/RBR6fgy76/). Ethical approval: CAAE: 73911617.2.0000.0071.
\end{abstract}

Keywords: Cartilage, Pain, Knee, Regenerative medicine, Cell therapy, Chondrocytes

\section{INTRODUCTION}

Articular cartilage injuries are a public health problem, which has become more and more frequent, affecting not only the elderly, but also the economically active young population. Some epidemiological studies have estimated that these injuries can be found in $61 \%$ of all performed knee arthroscopies. ${ }^{1-3}$
Although it does not always cause symptoms, chondral injury has two major problems in the short term, pain, joint effusion and movement blocks can impair performance in sports activities or in manual workers. In the long run, articular cartilage injuries are considered risk factors for the development of osteoarthritis, the most frequent cause of chronic pain and joint-related disability in the adult population. ${ }^{4-6}$ Articular cartilage is 
a tissue with a unique functional architecture, capable of supporting movements in multiple planes and with multiple load conditions, enabling movement without pain. However, trauma, illness or abnormal loading conditions can damage its structure. As it is a tissue without blood or lymphatic vessels and without nerves, the healing capacity of cartilage in adults is almost zero.

Currently available treatments are not good. When subchondral bone is also affected, bleeding could promote fibrocartilagenous tissue formation inside the lesion. $^{7-9}$ Based on this principle (perforating the subchondral bone and causing bleeding), several treatment methods have been proposed in the past. Together, all the techniques that use this principle are known as "bone marrow stimulation". In fact, one of these techniques, called "microfracture", is still the most widely used surgical method today to treat chondral lesions. ${ }^{10,11}$ But there are two major problems: the structural properties of fibrocartilage are different from those of hyaline cartilage, which can lead to degenerative changes in the subchondral bone over time, especially in young patients and athletes. Therefore, in theory microfracture does not prevent the development of osteoarthritis, but only relieves symptoms. In addition, perforation of the subchondral bone is an aggression in itself, with its consequences, such as the formation of intralesional osteophytes. Another consequence is that if the procedure is unsuccessful and the patient continues to have symptoms, the chance of successful revision surgery using cell therapy techniques will be greatly compromised. ${ }^{12-14}$

Another group of procedures emerged, based on the transfer of hyaline cartilage to cover the defect. This can be achieved with the transfer of autologous osteochondral cylinders (mosaicoplasty) removed from the injured knee itself. There are three problems: first, the number of cylinders is limited because of the small donor surface. Therefore, only small, single lesions can be treated in this way. ${ }^{15}$ Second, the removal of the cylinders causes an inflammatory response in the donor areas, which can trigger osteoarthritis. Third, this technique also injures the subchondral bone, with the consequences already mentioned above. ${ }^{8,13,14}$ Another way to transfer hyaline cartilage to the lesion is to use homologous transplants obtained from cadavers. This technique would be indicated for larger lesions, but it has serious drawbacks: the high financial cost and bureaucratic difficulty for the installation and maintenance of a musculoskeletal tissue bank; injury to the subchondral bone for graft fixation; the possibility of non-integration of the graft in the recipient's bone and the risk of transmission of infectious diseases. $^{11,16}$

Cell therapy could be the solution to treat cartilage lesions, specially the larger ones. In 1965, Chesterman et al was successful in isolating and expanding chondrocytes in vitro for the first time. ${ }^{17}$ In 1982, Peterson started an experimental model in rabbits, for the treatment of articular cartilage injuries. ${ }^{18}$ In 1984, the positive results of this model were presented in a medical congress, with $80 \%$ of the lesion being filled. ${ }^{19}$ In 1994 , the results of 23 patients operated on with this technique was published a high impact journal and started its worldwide use. ${ }^{20}$ The technique consists of obtaining a small fragment of articular cartilage from the patient and isolating the chondrocytes, which are the cells responsible for the manufacture of the extracellular matrix of the hyaline cartilage. It is this matrix that confers the special properties of this type of cartilage. Using cell culture techniques, the number of chondrocytes in the laboratory expands, which are then implanted back into the patient's knee.

In the early days, a piece of the patient's tibial periosteum needed to be removed, to be sutured over the lesion, creating a layer under which chondrocytes in liquid medium were injected, so that they would not spread through the joint. Several publications attest to the safety and effectiveness of this procedure, to the point that today, this technique is considered the gold standard for the treatment of chondral lesions with a diameter larger than two centimeters. ${ }^{21-24}$ The use of the periosteum has some drawbacks: the need for a second surgical incision on the tibia; handling difficulty due to retraction after withdrawal; graft delamination and loosening; graft hypertrophy. An important advance in the technique was the replacement of the periosteum with a collagen membrane. ${ }^{23,25-30}$ With the use of the membrane, the risk of re-operation for hypertrophy decreased from $25.7 \%$ to $5 \% .{ }^{31}$ In 2014, Saris et al published the results of the European multicenter study SUMMIT, in which the chondrocytes were placed inside the porcine membrane, a technique that became known as matrix-assisted chondrocyte implantation (MACI). ${ }^{32}$ MACI solved the problem of difficult sutures and won the microfracture in the SUMMIT study, showing that, at least in larger lesions, the chondrocyte is the gold standard treatment. In 2016, the FDA in the United States, authorized the commercial use of MACI. Since that, other countries and other similar products have been used in developed countries.

Autologous chondrocyte implantation using periosteum has already been successfully performed in patients in Brazil. ${ }^{33,34}$ The chondrocyte culture is already well established in our service, as attested by team members' publications. ${ }^{35-38}$ The concept was successfully tested in a large animal model. ${ }^{39}$ However, after a consultation to the Brazilian regulatory agency (ANVISA), it was requested that, before the locally produced membrane-assisted chondrocyte could be available for a larger scale clinical use, its safety and feasibility be tested.

\section{METHODS}

\section{Participants and setting}

The Hospital Albert Einstein's Research Ethics Committee approved this protocol (CAAE number 73911617.2.0000.0071). Orthopedic doctors of the 
hospital care team will inform patients about the research. Those who show interest in participating will be scheduled for an evaluation with one of the researchers, at which time more detailed information will be provided and the informed consent form will be explained to the patient.

\section{Inclusion criteria}

Male patients, aged between 18 and 45 years-old, with or without anterior cruciate ligament injury, who present with damage to the articular cartilage in any region of the distal femur or the patella, classified as grade 2 or 3 according to the International Cartilage Repair Society (ICRS) classification, diameter greater than $2 \mathrm{~cm}^{2}$, symptomatic and without improvement with conservative treatment based on physiotherapy, weight loss and medications.

\section{Exclusion criteria}

The exclusion criteria are superficial chondral lesion (ICRS grade 1 classification); subchondral bone injury (ICRS grade 4 classification); osteoarthritis (KellgrenLawrence score greater than 1); misalignment of the mechanical axis of the lower limb in the frontal plane; multiple ligament injuries; previous surgery on the affected knee; non-suturing meniscal injuries; systemic inflammatory diseases; inability to adhere to the rehabilitation protocol; functional limitations due to back or hip pain; claustrophobia; metallic implants that prevent magnetic resonance imaging; obesity (BMI greater than 30); smoking; cognitive changes with inability to read, understand and sign the informed consent.

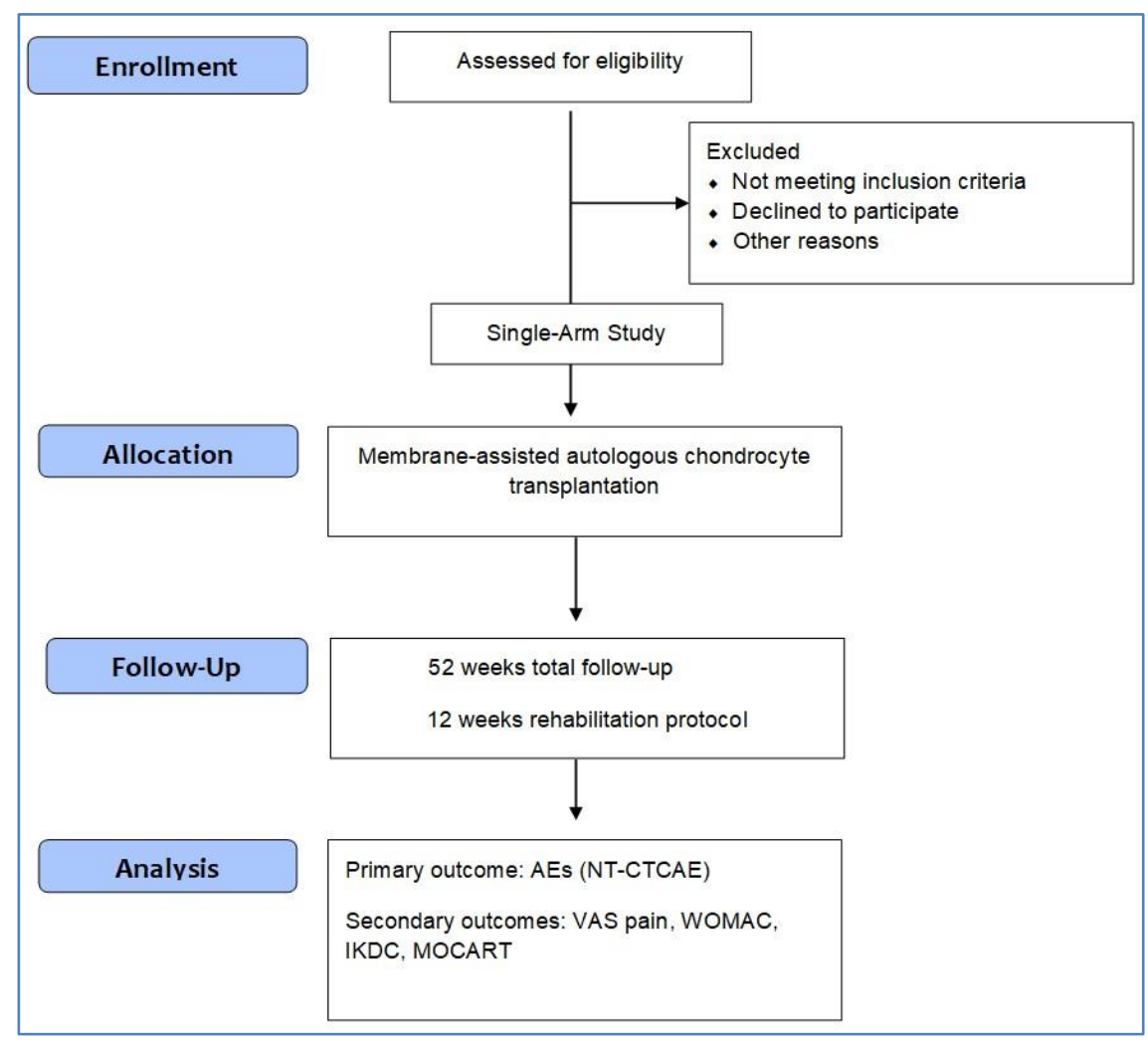

Figure 1: Flow chart of the participants through the study.

GMP=good manufacturing practices; VAS pain=verbal analogical scale of pain; WOMAC=Western-Ontario McMaster Universities Osteoarthritis Index; IKDC=International Knee Documentation Committee; MOCART=magnetic resonance observation of cartilage repair tissue.

\section{Interventions}

The study steps are summarized in Figure 1.

\section{First surgery}

\section{Arthroscopically assisted cartilage biopsy}

To obtain cartilage fragments, the patient will be submitted to arthroscopy of the injured knee under spinal anesthesia. Three fragments with five millimeters length (100 to $200 \mathrm{mg}$ ) will be collected from the articular cartilage in the intercondilar notch, with an arthroscopic open curette.

\section{Autologous human serum}

During the first surgery, $200 \mathrm{ml}$ of the patient's own blood will be collected in the operating room, in a bag without anticoagulant. This blood will be sent to the 
blood bank and the patient's autologous serum $(70 \mathrm{ml})$ is extracted. This serum will be added to the culture medium at a concentration of $20 \%$ to stimulate cell expansion.

\section{Chondrocyte isolation and expansion}

The cartilage fragments obtained from the biopsy will be transferred to the good manufacturing practices room at the cell therapy laboratory inside the hospital, in a sterile Falcon tube containing cold saline $(\mathrm{NaCl} 0.9 \%)$ and antibiotics (penicillin, streptomycin and amphotericin B, Gibco).

Chondrocyte isolation will be performed following the protocol previously described by Chesterman et al. ${ }^{17}$ Briefly, the cartilage will be fragmented and the fragments will be washed three times in culture medium containing: Ham's-F12 culture medium (Gibco) supplemented with $50 \mu \mathrm{g} / \mathrm{ml} \mathrm{L}$-ascorbic acid (Sigma), $1 \%$ antibiotic solution. antimycotic (penicillin, streptomycin and amphotericin B, Gibco), and 1\% L-glutamine solution (Gibco). Fragmented cartilage will be digested in collagenase type I solution (Sigma) for approximately 16 hours at $37{ }^{\circ} \mathrm{C}$. Thereafter, the cell suspension will be filtered using a $25 \mu \mathrm{m}$ diameter sterile pore filter, washed three times and the cells counted in the scepter (millipore) automated apparatus. Cells will be seeded in culture medium containing $20 \%$ autologous human serum at a density of 5,000 to 10,000 cells per $\mathrm{cm}^{2}$ in flasks of 25 or $75 \mathrm{~cm}^{2}$. The culture medium will be changed twice a week. When cells reach $80 \%$ confluence approximately 1 week after sowing, cells will be removed with trypsin or EDTA solution (Gibco) and seeded in new vials $\left(10,000\right.$ cells or $\left.\mathrm{cm}^{2}\right)$ to approximately $5 \times 10^{6}$ cells within 2 to 3 weeks.

\section{Cell seeding in the collagen membrane}

A commercially available collagen membrane will be used (Chondrogide®, Geistlich do Brasil, São Paulo, SP) as scaffold. The membrane will be washed with saline $(0.9 \% \mathrm{NaCl})$. Then $5 \times 10^{6}$ cells in culture medium will be sown on the membrane surface and incubated for 3 days for full interaction and cell-matrix adhesion. On the day of surgery, the membrane will be placed in a sealed sterile petri dish, in a thermal box with ice, to be sent to the operating room and implanted in the patient's knee. A membrane fragment will be sent to the laboratory to assess cell density and viability. Figure 2 illustrates some steps and the final appearance of the collagen matrix with chondrocytes.

\section{Second surgery}

\section{Autologous chondrocyte transplantation}

The surgical technique for performing the autologous chondrocyte implantation will be performed in the traditional way, already described and established in the literature. ${ }^{18,32}$ Briefly, in the operating room, the patient is placed in the supine position and anesthetized preferably with spinal block. If the anesthesiologist deems it necessary, general anesthesia may be required. A tourniquet is used at the root of the thigh of the limb to be operated, with individually adjusted size and pressure. Arthrotomy is performed via a medial or lateral meniscal access, depending on the affected condyle.
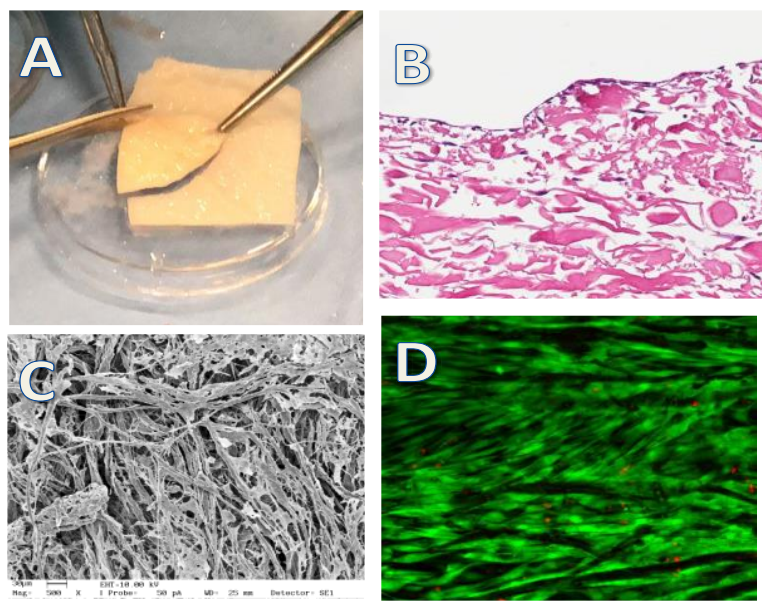

Figure 2: (A) Cell seeded collagen membrane (Chondrogide®, Geistlich do Brasil, São Paulo, SP) being cut with scissors and tweezers to adapt to the shape of the lesion; (B) conventional optical microscopy stained with $H \& E$ showing the membrane collagen fibers with attacked cells; (C) scanning electron microscopy; cell viability assay, (D) live/dead viability/cytotoxicity kit for mammalian cells

(Invitrogen, Carlsbad, CA, USA) demonstrating more than $90 \%$ cell viability after three weeks expansion plus seven days seeded inside the membrane.

The chondral lesion is debrided with a bone curette, until it reaches the subchondral bone at the base of the lesion, but without causing bleeding. Debridement at the margins must be carried out until healthy cartilage is reached, keeping the vertical edges. The defect must be modeled in a geometric shape. A sterile aluminum foil mold is used to create the exact shape and size of the lesion, which will be replicated on the collagen membrane. The membrane containing the chondrocytes is fixed in the lesion with fibrin glue (Tissucol, Baxter). According to surgeon judgment, if necessary, the membrane could be also sutured over the lesion, with three or four 5-0 absorbable suture stitches (Monocryl, Ethicon). The wound is closed in layers in the usual way and the incision is covered with a dressing. Drains will not be used. Immobilization will be used just for comfort if necessary in the first two weeks after surgery.

\section{Rehabilitation}

Patients will be guided and trained during hospitalization by a physiotherapist, to walk using two crutches. They will be maintained with knee immobilizer in extension for two weeks and without load for six weeks. They will 
be encouraged to practice active ankle movements, exercises to "awaken the quadriceps" with active isometric contractions. The goal is to maintain full knee extension in the first week and achieve 90 degree flexion in 6 weeks.

Hospital discharge will occur the day after surgery. The patient will have two physiotherapy sessions per week at the hospital, according to the following rehabilitation protocol: between the seventh and twelfth weeks after surgery, the load will progress to full load and the full range of motion. Between the thirteenth and the sixteenth week, strengthening, proprioception training and functional activities will be carried out. Full return to high-impact activities will only be allowed after one year of surgery, depending on the patient's symptoms and clinical tests.

\section{Outcomes}

Demographic data of patient and injury characteristics will be collected, such as age, gender, body mass index, and location of the lesion on MRI, lesion size, concomitant lesions. A complete clinical examination of gait and lower limbs will be performed before surgery and after one, two, three and six months of follow-up.

The safety of the procedure will be evaluated by the incidence of re-operations and the incidence and severity of complications according to version 4.0 of the list of the NCI-CTCAE (National Cancer Institute-Common Terminology Criteria for Adverse Events) (40).

Efficacy will be assessed through clinical scales and imaging tests: VAS pain (from 0 to 10); Western-Ontario McMaster Universities Osteoarthritis Index (WOMAC) clinical scale validated for the Portuguese language; subjective IKDC clinical scale; magnetic resonance observation of cartilage repair tissue (MOCART) magnetic resonance scale. ${ }^{41-43}$

\section{Follow-up}

As this is a pilot study, the follow-up time will be just one year, because most adverse events occur during this period. Table 1 summarizes follow-up visits.

\section{Statistical analysis}

Descriptive statistics will be used to analyze patient's demographics, clinical characteristics, cell culture, surgical features and outcomes. Categorical data will be presented as frequency and proportion. Continuous data will be presented as median and range. All statistical analysis will be performed using IBM SPSS version 22.0 (IBM Corp, Armonk, NY, USA).

\section{Data management and monitoring}

In this research, a data monitoring committee (DMC) will be not necessary due to the short duration of the study and low risks involved for the participants. The institutional review boards (IRBs) also have responsibility for monitoring the safety of trial participants at the institution. The data monitoring will be a liability of the three main investigators according to the institution. The access of the interim results will be a duty of the main and second investigators that includes the decision to terminate the trial in any stage of the study. Any adverse event will be reported to the researchers involved and communicated to the main investigator according to the IRBs description.

Table 1: Study follow-up.

\begin{tabular}{|lll|}
\hline Visit & Time & Purpose \\
\hline $\mathbf{1}$ & Pre op & Enrollment \\
\hline $\mathbf{2}$ & Pre op & Blood collection \\
\hline $\mathbf{4}$ & Pre op & $\begin{array}{l}\text { Arthroscopically assisted } \\
\text { cartilage biopsy }\end{array}$ \\
\hline $\mathbf{5}$ & Day 0 & MACI \\
\hline $\mathbf{6}$ & 2 weeks & $\begin{array}{l}\text { AEs, VAS pain, start } \\
\text { rehabilitation protocol }\end{array}$ \\
\hline $\mathbf{7}$ & 4 weeks & AEs, VAS pain \\
\hline $\mathbf{8}$ & 12 weeks & $\begin{array}{l}\text { AEs, IKDC, WOin } \\
\text { rehabilitation protocol }\end{array}$ \\
\hline $\mathbf{9}$ & 26 weeks & AEs, IKDC, WOMAC \\
\hline $\mathbf{1 0}$ & 52 weeks & $\begin{array}{l}\text { AEs, IKDC, WOMAC and } \\
\text { MOCART (MRI) }\end{array}$ \\
\hline
\end{tabular}

$\mathrm{AES}=$ adverse events; $\mathrm{PRE}-\mathrm{OP}=$ pre-operative visit; VAS PAIN=verbal analogical scale of pain; WOMAC=WesternOntario Mcmaster Universities Osteoarthritis Index; IKDC=International Knee Documentation Committee; MACI= Matrix-Assisted Chondrocyte Implantation; MOCART= magnetic resonance observation of cartilage repair tissue; MRI=magnetic resonance image.

\section{DISCUSSION}

This protocol arose from the need to obtain authorization from the Brazilian regulatory agency (ANVISA) for the clinical use of an advanced cell therapy product already established and used routinely in developed countries in North America, Europe and Asia. The autologous chondrocyte, the active ingredient of this technique, is a cell obtained from the patient himself. However, the process for isolation, expansion and seeding on the collagen membrane (scaffold) may vary according to the manufacturer. Currently in Latin America, no company provides this service. For this reason, ANVISA requested that a feasibility and safety study be carried out.

Another concern regarding the use of chondrocytes in a developing country is the issue of high production costs. There is a fear that the therapy will not be cost-effective. However, studies suggest that chondrocyte transplantation, by preventing the progression of chondral lesions in young individuals for severe osteoarthritis and knee arthroplasty in old age, may be an advantageous strategy even for universal public health systems, such as 
SUS (Sistema Único de Saúde) in Brazil. ${ }^{44}$ Currently, the queues for performing hip and knee arthroplasties are the longest among all surgeries offered at SUS, and it takes more than five years for a patient to be operated. Bearing in mind that many Brazilian health insurances already offer reimbursement for the treatment of cartilage lesions with collagen membranes, we believe it is possible to offer the option with cells within a viable price, since our protocol uses relatively cheap inputs for cell expansion.

This protocol has some limitations. Due to the high cost to develop clinical studies with advanced cell therapy products, the institution chose, in agreement with ANVISA, to carry out this first feasibility study in a very small sample of only three participants. This study is unable to verify the product's effectiveness, since the sample is small and there is no control group. In the future, the data obtained and lessons learned will be applied to develop larger phase $2 / 3$ studies to verify the effectiveness of the product.

\section{CONCLUSION}

Matrix-assisted autologous chondrocyte transplantation is the gold standard treatment for larger articular cartilage lesions. However, there is currently no product of this type enabled for clinical use in Brazil. This feasibility and safety phase 1 study will serve as the base for a future pre-market phase $2 / 3$ randomized clinical trial, to introduce the first advanced cell therapy product for articular cartilage treatment in Brazil.

Funding: Hospital Israelita Albert Einstein (SGPP 311417) Conflict of interest: None declared

Ethical approval: IRB of Hospital Israelita Albert Einstein (CAAE $n^{\circ}$ 73911617.2.0000.0071)

\section{REFERENCES}

1. Hjelle K, Solheim E, Strand T, Muri R, Brittberg M. Articular cartilage defects in 1,000 knee arthroscopies. Arthroscopy: J Arthroscopic Related Surg. 2002;18(7):730-4.

2. Widuchowski W, Widuchowski J, Trzaska T. Articular cartilage defects: Study of 25,124 knee arthroscopies. Knee. 2007;14(3):177-82.

3. Curl WW, Krome J, Gordon S, Rushing J, Smith BP, Poehling GG. Cartilage Injuries: A Review of 31,516 Knee Arthroscopies. Arthroscopy: J Arthroscopic Related Surg. 1997;13(4):456-60.

4. Widuchowski W, Widuchowski J, Faltus R, Lukasik P, Kwiatkowski G, Szyluk K, et al. Long-term clinical and radiological assessment of untreated severe cartilage damage in the knee: a natural history study. Scandinavian J Med Sci Sports. 2011;21(1):106-10.

5. Widuchowski W, Widuchowski J, Koczy B, Szyluk K. Untreated Asymptomatic Deep Cartilage Lesions Associated With Anterior Cruciate Ligament Injury:
Results at 10- and 15-Year Follow-Up. Am J Sports Med. 2009;37(4):688-92.

6. Figueroa D, Calvo R, Vaisman A, Carrasco MA, Moraga C, Delgado I. Knee Chondral Lesions: Incidence and Correlation Between Arthroscopic and Magnetic Resonance Findings. Arthroscopy: J Arthroscopic Related Surg. 2007;23(3):312-5.

7. Nicolini AP, Carvalho RT, Dragone B, Lenza M, Cohen M, Ferretti M. Updates in biological therapies for knee injuries: full thickness cartilage defect. Curr Rev Musculoskelet Med. 2014;7(3):256-62.

8. Gomoll AH, Farr J, Gillogly SD, Kercher J, Minas T. Surgical management of articular cartilage defects of the knee. J Bone Joint Surg Am. 2010;92:2470-90.

9. Steadman JR, Rodkey WG, Singleton SB. Microfracture technique forfull-thickness chondral defects: Technique and clinical results. Operative Techniques, 1997.

10. Solheim E, Hegna J, Inderhaug E, Oyen J, Harlem T, Strand T. Results at 10-14 years after microfracture treatment of articular cartilage defects in the knee. Knee Surg Sports Traumatol Arthrosc. Springer Berlin Heidelberg; 2014: 1-7.

11. Montgomery SR, Foster BD, Ngo SS, Terrell RD, Wang JC, Petrigliano FA, et al. Trends in the surgical treatment of articular cartilage defects of the knee in the United States. Knee Surg Sports Traumatol Arthrosc. Springer Berlin Heidelberg; 2014;22(9):2070-5.

12. Gomoll AH, Minas T. The quality of healing: articular cartilage. Wound Repair Regen. 2014;22 Suppl 1(S1):30-8.

13. Gomoll AH, Madry H, Knutsen G, van Dijk N, Seil $\mathrm{R}$, Brittberg $\mathrm{M}$, et al. The subchondral bone in articular cartilage repair: current problems in the surgical management. Knee Surg Sports Traumatol Arthrosc. 2010;18(4):434-47.

14. Minas T, Gomoll AH, Rosenberger R, Royce RO, Bryant $T$. Increased failure rate of autologous chondrocyte implantation after previous treatment with marrow stimulation techniques. Am J Sports Med. 2009;37(5):902-8.

15. Lane JG, Healey RM, Chen AC, Sah RL, Amiel D. Can Osteochondral Grafting Be Augmented With Microfracture in an Extended-Size Lesion of Articular Cartilage? Am J Sports Med. 2010;38(7):1316-23.

16. Camp CL, Stuart MJ, Krych AJ. Current concepts of articular cartilage restoration techniques in the knee. Sports Health. Sage Publications; 2014;6(3):265-73.

17. Chesterman PJ, Smith AU. Homotransplantation of articular cartilage and isolated chondrocytes. JBJS. 1968;50(1):184-197.

18. Peterson L, Menche D, Grande D. Chondrocyte transplantation. An experimental model in the rabbit. In: Transactions from the 30th annual Orthopedic Research Society, Atlanta: 1984. 
19. Grande DA, Pitman MI, Peterson L, Menche, D. and Klein, M. (1989), The repair of experimentally produced defects in rabbit articular cartilage by autologous chondrocyte transplantation. J. Orthop. Res. 1989;7:208-18.

20. Brittberg M, Lindahl A, Nilsson A, Ohlsson C, Isaksson O, Peterson L. Treatment of deep cartilage defects in the knee with autologous chondrocyte transplantation. N Engl J Med. 1994;331(14):88995.

21. Rosenberger RE, Gomoll AH, Bryant T, Minas T. Repair of large chondral defects of the knee with autologous chondrocyte implantation in patients 45 years or older. Am Orthop Society Sports Med. 2008;36(12):2336-44.

22. Oussedik S, Tsitskaris K, Parker D. Treatment of Articular Cartilage Lesions of the Knee by Microfracture or Autologous Chondrocyte Implantation: A Systematic Review. Arthroscopy. 2013;(S1):S3-S10.

23. Jacobi M, Villa V, Magnussen RA, Neyret P. MACI - a new era? Sports Medicine, Arthroscopy, Rehabilitation, Therapy \& Technology. BioMed Central Ltd; 2011;3(1):10.

24. Harris JD, Hussey K, Wilson H, Pilz K, Gupta AK, Gomoll A, et al. Biological knee reconstruction for combined malalignment, meniscal deficiency, and articular cartilage disease. Arthroscopy. 2015;31(2):275-82.

25. Zheng M-H, Willers C, Kirilak L, Yates P, Xu J, Wood D, et al. Matrix-Induced Autologous Chondrocyte Implantation (MACI $®)$ ): Biological and Histological Assessment. Tissue Engineering. 2007;13(4):737-46.

26. Bartlett W. Autologous chondrocyte implantation versus matrix-induced autologous chondrocyte implantation for osteochondral defects of the knee: A Prospective, Randomised Study. Br Editorial Society Bone Joint Surg. 2005;87(5):640-5.

27. Saris D, Price A, Widuchowski W, BertrandMarchand M, Caron J, Drogset JO, et al. MatrixApplied Characterized Autologous Cultured Chondrocytes Versus Microfracture: Two-Year Follow-up of a Prospective Randomized Trial. Am Orthop Society Sports Med. 2014;42(6):1384-94.

28. Herbort M, Zelle S, Rosenbaum D, Osada N, Raschke M, Petersen W, et al. Arthroscopic Fixation of Matrix-Associated Autologous Chondrocyte Implantation: Importance of Fixation Pin Angle on Joint Compression Forces. YJARS. Elsevier Inc; 2011;27(6):809-16.

29. Ebert JR, Robertson WB, Woodhouse J, Fallon M, Zheng MH, Ackland T, et al. Clinical and Magnetic Resonance Imaging-Based Outcomes to 5 Years After Matrix-Induced Autologous Chondrocyte Implantation to Address Articular Cartilage Defects in the Knee. Am J Sports Med. 2011;39(4):753-63.

30. Marlovits S, Aldrian S, Wondrasch B, Zak L, Albrecht C, Welsch G, et al. Clinical and Radiological Outcomes 5 Years After Matrix-
Induced Autologous Chondrocyte Implantation in Patients With Symptomatic, Traumatic Chondral Defects. Am J Sports Med. 2012;40(10):2273-80.

31. Gomoll AH, Probst C, Farr J, Cole BJ, Minas T. Use of a Type I/III Bilayer Collagen Membrane Decreases Reoperation Rates for Symptomatic Hypertrophy After Autologous Chondrocyte Implantation. Am J Sports Med. 2009;37(1):20-3.

32. Saris D, Price A, Widuchowski W, BertrandMarchand M, Caron J, Drogset JO, et al. MatrixApplied Characterized Autologous Cultured Chondrocytes Versus Microfracture: Two-Year Follow-up of a Prospective Randomized Trial. Am J Sports Med. Am Orthop Society Sports Med. 2014;42(6):1384-94.

33. Cohen M, Nery C, Peccin MS, Ressio CR. Autologous chondrocyte implantation to treat femoral condyle and talar lesions. Einstein. 2008;6(1):37-41.

34. Lombello C, Reis GM, Cohen M. Study on human chondrocyte culture viability for autologous transplantation in clinical application. Einstein. 2003; $1: 84-8$.

35. Antonioli E, Piccinato CA, Nader HB, Cohen M, Goldberg AC, Ferretti M. Modulation of Hyaluronan Synthesis by the Interaction between Mesenchymal Stem Cells and Osteoarthritic Chondrocytes. Stem Cells International. Hindawi Publishing Corporation; 2015;2015(2):640218-11.

36. Antonioli E, Lobo AO, Ferretti M, Cohen M, Marciano FR, Corat EJ, et al. An evaluation of chondrocyte morphology and gene expression on superhydrophilic vertically-aligned multi-walled carbon nanotube films. Mater Sci Eng C Mater Biol Appl. 2013;33(2):641-7.

37. Pintan GF, de Oliveira AS, Lenza M, Antonioli E, Ferretti M. Update on biological therapies for knee injuries: osteoarthritis. Curr Rev Musculoskelet Med. Springer US; 2014;7(3):263-9.

38. da Cunha Cavalcanti FMM, Doca D, Cohen M, Ferretti M. Updating on diagnosis and treatment of chondral lesion of the knee. Revista Brasileira de Ortopedia. 2012;47(1):12-20.

39. Zorzi A, Amstalden E, Plepis A, Martins V, Ferretti M, Antonioli E, et al. Effect of human adipose tissue mesenchymal stem cells on the regeneration of ovine articular cartilage. Int $\mathrm{J}$ Mol Sci. 2015;16(11):26813-31.

40. National Cancer Institute. Common Terminology Criteria for Adverse Events (CTCAE). 2010: 1-80.

41. Fernandes MI. Tradução e validação do questionário de qualidade de vida específico para osteoartrose WOMAC (Western Ontário McMaster Universities) para a língua portuguesa. [dissertação]. São Paulo: Universidade Federal de São Paulo, Escola Paulista de Medicina; 2003.

42. Metsavaht L, Leporace G, Riberto M, de Mello Sposito MM, Batista LA. Translation and CrossCultural Adaptation of the Brazilian Version of the International Knee Documentation Committee 
Subjective Knee Form: Validity and Reproducibility. Am J Sports Med. 2010;38(9):1894-9.

43. Marlovits S, Singer P, Zeller P, Mandl I, Haller J, Trattnig S. Magnetic resonance observation of cartilage repair tissue (MOCART) for the evaluation of autologous chondrocyte transplantation: Determination of interobserver variability and correlation to clinical outcome after 2 years. European J Radiol. 2006;57(1):16-23.

44. Martín AR, Patel JM, Zlotnik HM, Carey JL, Mauck RL. Emerging therapies for cartilage regeneration in current excluded "red knee" populations. NPJ Regen Med. 2019;4:12.

Cite this article as: Zorzi AR, Antonioli E, Cohen M, Kaleka CC, Kondo AT, Kutner JM, et al. Autologous chondrocyte transplantation: a phase 1 study protocol to validate the safety and feasibility of a new advanced cell therapy product for articular cartilage repair in Brazil. Int J Clin Trials 2020;7(2):123-30. 